\title{
Metalloid Reductase of Pseudomonas moravenis Stanleyae Conveys Nanoparticle Mediated Metalloid Tolerance
}

\author{
Richard Nemeth, Mackenzie Neubert, ${ }^{\dagger}$ Zachary J. Butz, Thomas W. Ni, ${ }^{\dagger}$ and Christopher J. Ackerson*(1) \\ Department of Chemistry, Colorado State University, Fort Collins, Colorado 80523, United States \\ Supporting Information
}

ABSTRACT: A glutathione reductase (GSHR)-like enzyme in Pseudomonas moraviensis stanleyae was previously implicated as underlying the bacterium's remarkable $\mathrm{SeO}_{3}{ }^{2-}$ tolerance. Herein, this enzyme is sequenced, recombinantly expressed, and fully characterized. The enzyme is highly adapted for selenodiglutathione substrates $\left(K_{\mathrm{m}}=336 \mu \mathrm{M}\right)$ compared to oxidized glutathione $\left(K_{\mathrm{m}}=8.22 \mathrm{mM}\right)$. The recombinant expression of this enzyme in the laboratory strains of Escherichia coli conveys a 10 -fold increase in $\mathrm{IC}_{90}$ for $\mathrm{SeO}_{3}{ }^{2-}$. Moreover, selenium nanoparticles are observed when the enzyme is overexpressed in the cells exposed to $\mathrm{SeO}_{3}{ }^{2-}$, but not in the corresponding noenzyme controls. The analyses of the structural homology models of the enzyme reveal changes in the parts of the enzyme associated with product release, which may underlie the Se substrate specialization. Combined, the observations of adaptation to Se reduction over oxidized glutathione reduction as well as the portability of this nanoparticle-mediated $\mathrm{SeO}_{3}{ }^{2-}$ tolerance into other cell lines suggest that the P. moraviensis GSHR may be better described as a GSHR-like metalloid reductase.

\section{INTRODUCTION}

The enzymatic conversion of soluble inorganic ions into insoluble forms is accomplished by enzyme centers such as those found in ferritin, magnetosomes, and silicateins. This enzymatic alteration in the solubility state facilitates the synthesis of biogenic inorganic materials. ${ }^{1-3}$ These naturally occurring catalysts, in concert with accessory proteins, can exhibit control over subsequent material composition, oxidation state, morphology, and structure. These natural precedents suggest that the intentional engineering of biological diversity could underlie the engineered diversity in biogenically synthesized inorganic materials. Such inorganic materials - synthesized by laboratory-evolved or engineered biomolecules (peptides, proteins, and nucleic acids) attract attention in catalysis, self-assembly, and in biocontrast (labeling) applications. ${ }^{4-8}$

Many self-contained biological systems, for synthesizing an inorganic nanostructure, will generally require an oxidoreductase activity, enabling the conversion of inorganic ions from soluble to insoluble oxidation states. Ferritins and DNAbinding proteins accomplish this with the ferroxidase enzymatic centers. ${ }^{9,10}$ Mercuric reductases accomplish this with substrate reduction through an active dithiol, ${ }^{11}$ which exhibits striking similarities to other enzymes such as lipoamide dehydrogenase. $^{12}$

We recently reported on the ability of glutathione reductase (GSHR) to enzymatically reduce selenite $\left(\mathrm{SeO}_{3}{ }^{2-}\right)$ to zerovalent red selenium in a nicotinamide adenine dinucleotide phosphate (NADPH)-dependent reaction. ${ }^{13}$ Similar, although diminished, activity was observed for the same enzyme in reducing tellurite $\left(\mathrm{TeO}_{3}{ }^{2-}\right)$ to elemental Te. Our prior work identified the selenite reductase activity in Pseudomonas moravenis stanleyae. This microbe attracted our attention because it is found as an endophyte in the selenium-tolerant plant, Stanleya pinnata. When cultured independently in liquid media, it tolerates $\mathrm{SeO}_{3}{ }^{2-}$ supplementation in liquid culture up to $10 \mathrm{mM}$. This is 10 -fold more than the $\mathrm{SeO}_{3}{ }^{2-}$ tolerance of most other microbes. We attributed the observed selenite reduction activity of $P$. moravenis to a GSHR-like enzyme on the basis of proteomic mass spectrometry of an in-gel in situ selenium reductase activity.

GSHRs generally belong to the family of pyridine nucleoside-dependent oxidoreductases. This enzyme family also, notably, includes another well-characterized metal-reducing enzyme-mercuric reductase. ${ }^{14}$ Within this enzyme family, the active sites are highly conserved. The typical active site peptide sequences are CXXXXC for type I and CXXC for type II enzymes. ${ }^{15}$ These classes of enzymes have demonstrated their ability to reduce a variety of metal ions to zerovalent forms, including $\mathrm{Se}, \mathrm{Hg}, \mathrm{Te}, \mathrm{Fe}, \mathrm{Cr}$, and $\mathrm{U}^{16-19}$ GSHR is reported to reduce $\mathrm{Au}$ (III) to the zerovalent form as well. ${ }^{20}$ Thus, the class of pyridine nucleoside-dependent oxidoreductases may repre-

Received: July 13, 2018

Accepted: August 17, 2018

Published: November 5, 2018 
Table 1. Sequence and Structural Homology ${ }^{a}$

$\begin{array}{lcccc} & \text { PM MTLR } & \text { PF GSHR } & \text { EC GSHR } & \text { SC GSHR } \\ \text { PM MTLR } & & 93.10 \% / 1.56 & 74.14 \% / 1.23 & 60.78 \% * / 1.27 \\ \text { PF GSHR } & 98.00 \% / 1.71 & & 77.59 \% / 1.05 & 75.47 \% * / 1.12 \\ \text { EC GSHR } & 67.42 \% / 1.44 & 68.31 \% / 1.42 & & 81.48 \% * / 0.87 \\ \text { SC GHSR } & 70.92 \% / 1.56 & 73.48 \% / 1.33 & 74.61 \% / 1.17 & 79.63 \% * / 0.85 \\ \text { HS GSHR } & 70.34 \% / 1.44 & 70.63 \% / 1.24 & 78.97 \% / 1.07 & 84.91 \% / 0.56 \\ \end{array}$

${ }^{a}$ Sequence similarity (left value) and RMSD calculations (right values). PF--P. fluoresceine, EC--E. coli , SC--S. cervisiae, and HS--H. sapiens.
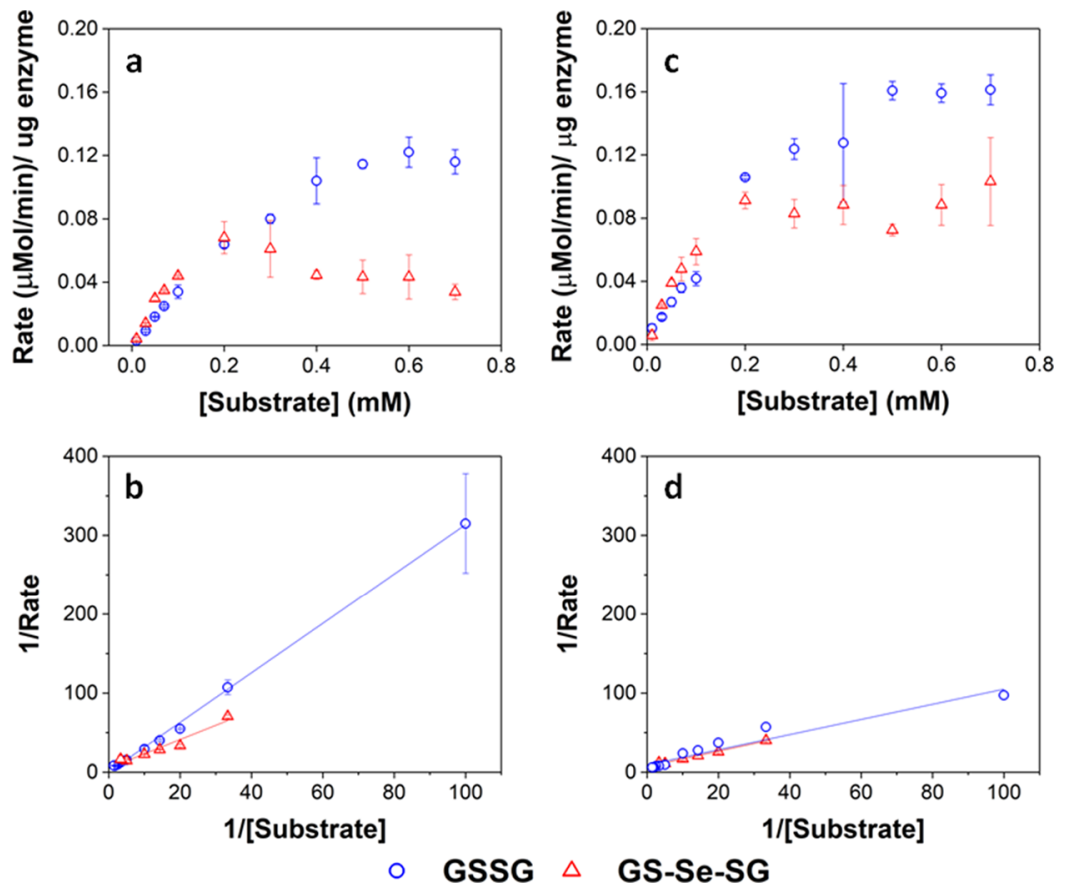

Figure 1. Substrate activity assays: (a) GRLMR, (c) GSHR, with the corresponding Lineweaver-Burke plots: (b) GRLMR. (d) GSHR.

sent an evolutionarily adaptable platform of inorganic ion reductases, with modifications to the enzyme, altering the metal-ion selectivity. Such a catalytic center, with alterable precursor selectivity, is of interest in biogenic inorganic nanoparticle (NP) synthesis.

In a prior study, we characterized the commercially sourced Saccharomyces cervisiae GSHR for selenite reductase activity, showing the ability of the enzyme to oxidize NADPH while reducing $\mathrm{SeO}_{3}{ }^{2-}$ to $\mathrm{Se}(0) \mathrm{NPs}^{13}$ In the present study, we characterize a homologous metalloid reductase from the seleno-specialist $P$. moraviensis. We find that the substrate selectivity of the metalloid reductase $\left(K_{\mathrm{m}}\right)$ shows a substantially larger preference for GS-Se-SG relative to all other reported GSHR enzymes. These enzymatic properties can be partially rationalized in terms of sequence and the corresponding homology-modeled structure of the enzyme. We also observe that expressing this enzyme in the laboratory strains of E. coli (BL21, SS320) results in an increased tolerance to $\mathrm{SeO}_{3}{ }^{2-}$, as well as the presence of Se NPs in these cells. Overall, our data suggest that the enzyme may be best described as a GSHR-like metalloid reductase (GRLMR).

\section{RESULTS AND DISCUSSION}

The altered substrate specificity of GRLMR enzymes, favoring selenodiglutathione (GS-Se-SG) over oxidized glutathione (GSSG) as a substrate, could underlie the remarkable $\mathrm{SeO}_{3}{ }^{2-}$ tolerance of $P$. moravenis stanleyae. We therefore characterized the $P$. moravenis stanleyae GRLMR enzyme identified previously. The DNA sequence of the enzyme was acquired through a full-genome sequencing (ACGT Inc., Wheeling, IL). The sequencing was conducted using de novo paired-end sequencing. ${ }^{21}$ This revealed a genome in which $70.3 \%$ of the nucleobases have, at the most, a 1:1000 probability of misassignment. Figure S1 shows the "Quality Score" ( $Q$ score) for each sequenced base with $Q=-\log$ 10(e). The $Q$ scores are derived from a phred-like error probability assessment of each individual nucleotide. ${ }^{22}$

A basic local alignment search tool search of the genomic sequence, using the Pseudomonas R-28S GSHR as a reference, identified one GSHR-like sequence, with 93\% sequence homology. The sequence alignment of this GRLMR DNA using Serial Cloner shows a high similarity (98.00\%) to Pseudomonas fluorescines GSHR and modest similarity (67$71 \%)$ to Escherichia coli, Stanleya cervisiae, and Homo sapiens GSHR DNA. The sequence similarities are summarized in Table 1, and full alignments are shown in the Supporting Information, Figure S2. The DNA sequence, combined with the homology modeling of the structure, suggests that all of the structural domains of type I pyridine nucleotide-dependent oxidoreductases, including catalysis, dimerization, nucleotide binding, and substrate/product-binding domains, ${ }^{15,23,24}$ are present in this enzyme. Such a homology would suggest that the GRLMR enzyme would conduct substrate reduction for GSSG, and related molecules, in a fashion similar to previously 
reported GSHR reduction pathways via an active-site dithiol. $^{25,26}$ This GRLMR shows 19\% DNA sequence homology to mercuric reductase from Pseudomonas aeruginosa.

Homology modeling using intensive parameters on the Phyre 2 server $^{27}$ suggests that the structures of the Pseudomonas-derived GSHR and GRLMR enzymes are homologous to the other GSHR enzymes, despite modest DNA sequence divergence. Table 1 shows the root-meansquare deviation (RMSD) of the atomic position values for a set of GSHR homology models and/or crystal structures. Overall, the RMSD values for these structures are similar, suggesting an overall structural homology between GRLMR, and GSHR from P. fluorescines, E. coli, H. sapiens, and S. cervisiae.

Under the hypothesis that the GRLMR enzyme has altered selectivity relative to other characterized GSHRs, we characterized the enzyme kinetics of both GRLMR and $S$. cervisiae GSHR. We expressed GRLMR recombinantly in the E. coli BL21 cells. Following a $6 \times$-histidine tag purification, we determined the Michaelis-Menton constants $\left(K_{\mathrm{m}}\right)$ for both GRLMR and the commercially sourced $S$. cervisiae GSHR (Sigma, G3664). The $K_{\mathrm{m}}$ value of each enzyme was determined for each of the three substrates: $\mathrm{SeO}_{3}{ }^{2-}$, GSSG, and GS-Se-SG. It has been previously reported that the GSHR enzymes have the ability to reduce these three substrates, ${ }^{13}$ with the proposed mechanisms for the GSH-based substrates. ${ }^{25,26}$ While $\mathrm{SeO}_{3}{ }^{2-}$ (Alfa Aesar, 12585) and GSSG (Sigma, G4376) are commercially available, we synthesized GS-Se-SG according to the previous published methods. ${ }^{26}$

The enzymatic rates for both enzymes with both the GSSG and GS-Se-SG substrates are plotted in Figure 1, panels $(\mathrm{a}, \mathrm{c})$. The data are plotted as NADPH cofactor consumption, observed experimentally as the depletion of a spectroscopic peak characteristic of NADPH (but not $\mathrm{NADP}^{+}$) at $340 \mathrm{~nm}$. The decay rate, as measured at $340 \mathrm{~nm}$, was converted to a normalized reaction rate. Lineweaver-Burke plots (shown as insets) were generated for each enzyme to determine $V_{\max }$ and $K_{\mathrm{m}}$ for the corresponding substrates. We chose to utilize the Lineweaver-Burke analysis over the more current method of nonlinear regression because our data was not accurately modeled using this technique. Points after $0.2 \mathrm{mM}$ for GS-SeSG were not used for the determination of $K_{\mathrm{m}}$ because of particle scattering altering the observed rate. Without having the data points nearing the asymptote, the nonlinear regression analysis suffered from large errors and major variations in the calculated constants. When nonlinear regression was used for the analysis of all GSSG data points, the reciprocal plots showed an uneven distribution, indicating that the enzyme kinetics were not modeled correctly using this method. Upon further investigation, we discovered that the reported $K_{\mathrm{m}}$ values for SC GSHR were calculated using the LineweaverBurke analysis. ${ }^{28}$ Using the Lineweaver-Burke analysis granted us consistency across our data analysis and allowed for comparison with the literature, giving us the most accurate and comparable values relative to the other common kinetic analyses. The determined $K_{\mathrm{m}}$ values for each enzyme/substrate combination are shown in Table 2 .

For the GRLMR enzyme, we observe a remarkable specialization of the enzyme for GS-Se-SG over GSSG. Specifically, the $K_{\mathrm{m}}$ value of GRLMR is 25 times more favorable for GS-Se-SG as compared to GSSG. This difference in $K_{\mathrm{m}}$ for the two substrates strongly implies that the enzyme is specialized for Se reduction over GSSG reduction. For $S$.
Table 2. Enzyme Kinetics ${ }^{a}$

$\begin{array}{ccccc}\text { enzyme/substrate } & \begin{array}{c}\text { GRLMR } \\ K_{\mathrm{m}}\end{array} & \begin{array}{c}\text { SC GSHR } \\ (\mu \mathrm{M})\end{array} & \begin{array}{c}\text { GRLMR } \\ V_{\max }\end{array} & \begin{array}{c}\text { SC GSHR } \\ V_{\max }\end{array} \\ \text { GSSG } & 8.22 \mathrm{mM} & 103 & 2.62 & 0.107 \\ \text { GS-Se-SG } & 336 \mu \mathrm{M} & 133 & 0.187 & 0.137 \\ { }^{a} V_{\max } \text { reported in } & (\mu \mathrm{M} / \mathrm{min}) /(\mu \mathrm{g} \text { of enzyme). } & & \\ \end{array}$

cervisiae GSHR, we determined similar $K_{\mathrm{m}}$ values for GSSG and GS-Se-SG substrates, with the enzyme showing a slightly greater affinity for GSSG. The values we find for both substrates are consistent with the previous findings from other research groups. ${ }^{13,28,28}$ The $K_{\mathrm{m}}$ value of GRLMR for $\mathrm{SeO}_{3}{ }^{2-}$ has not been previously reported, to our knowledge. The physiological relevance of the $K_{\mathrm{m}}$ value for $\mathrm{SeO}_{3}{ }^{2-}$ is questionable, as $\mathrm{Se}$ salts such as $\mathrm{SeO}_{3}{ }^{2-}$ are converted to GS-Se-SG via GSH reduction in vivo. ${ }^{29}$ We established here the $K_{\mathrm{m}}$ value for $\mathrm{SeO}_{3}{ }^{2-}$ for both GRLMR and S. cervisiae GSHR because we identified GRLMR on the basis of the $\mathrm{SeO}_{3}{ }^{2-}$ reductase activity, but note that the value is sufficiently unfavorable compared to the GSH-based substrates that it is unlikely to be physiologically relevant.

The value of $V_{\max }$ for the GS-SG and GS-Se-SG substrates track in tandem up to the initial concentrations of $0.20 \mathrm{mM}$. Deviations begin near a concentration of $0.30 \mathrm{mM}$, where the rate for GS-Se-SG has reached its maximum velocity, whereas the rate for oxidized glutathione continues to increase until roughly $0.60 \mathrm{mM}$ substrate concentration. The maximum velocity observed for GSSG is approximately 14 times greater than the $V_{\max }$ for GS-Se-SG, but this occurs at a larger substrate concentration for GSSG, accounting for the lower affinity. It is possible that the fall-off in rate that we observe at higher GSSe-SG concentrations is artifactual, arising from the interference in the optical assay by the selenium NPs produced during the experiment.

Overall, the $K_{\mathrm{m}}$ values we find suggest a strong substrate preference for GS-Se-SG for the GRLMR enzyme. This is in contrast to the other characterized GSHR enzymes, where there is essentially no differentiation between the substrates. The cellular concentrations of GSSG would typically be much higher than GS-Se-SG at sublethal amounts of selenium in vivo; thus, the SC GSHR enzyme would turn over GSSG much more frequently and does not lead to selenium resistance as in the P. Moraviensis system. To our knowledge, this is the first finding of an enzyme specialized for reduction of GS-Se-SG over any other substrate. Such a specialized enzyme could be used for nanomaterial development such as biogenic quantum dots and, most notably, as a starting point for the development of a functionalized clonable NP.

We hypothesized that if GRLMR conveys $\mathrm{SeO}_{3}{ }^{2-}$ tolerance to $P$. moraviensis, then the recombinant expression of this enzyme may convey a similar tolerance to the host organism for GRLMR expression. To evaluate this hypothesis, we transformed the lab expression strains of E. coli, BL21 and SS320, with the isopropyl $\beta$-D-1-thiogalactopyranoside (IPTG)-inducible expression vector described above. For the GRLMR enzyme-expressing $E$. coli, we determined selenite tolerance as the concentration of $\mathrm{SeO}_{3}{ }^{2-}$ that kills $90 \%$ of the cells $\left(\mathrm{IC}_{90}\right)$. We also examined the cells microscopically for the presence or absence of SeNPs.

For $\mathrm{IC}_{90}$ determination, identical volumes of cells were plated on lysogeny broth (LB) agar and $\mathrm{LB}$ agar $+\mathrm{SeO}_{3}{ }^{2-}$, each in triplicate, and grown overnight at $37^{\circ} \mathrm{C}$. The following 

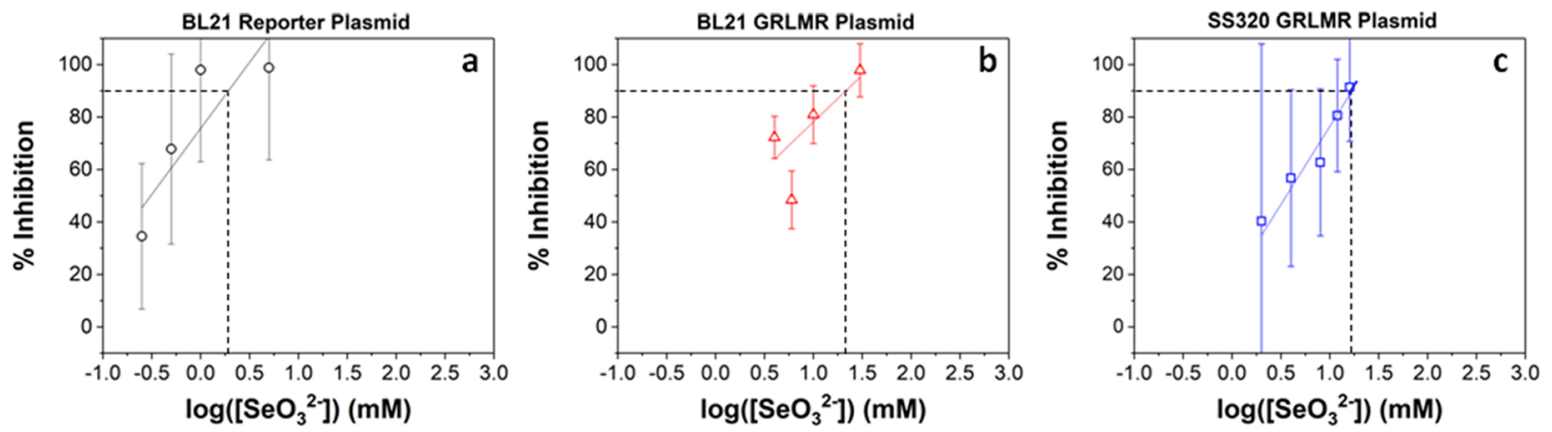

Figure 2. $\mathrm{IC}_{90}$ assays reported in percent inhibition vs selenite concentration: (a) BL21 E. coli cell lines with a GFP plasmid; (b) BL21 cells with the GRLMR plasmid; and (c) SS320 cells with the GRLMR plasmid.

day, the colony-forming units (cfus) were counted. $\mathrm{IC}_{90}$ was calculated as the percentage of cfus present relative to an identical control supplemented with $1.0 \mu \mathrm{M} \mathrm{SeO}{ }_{3}{ }^{2-}$. We determined $1.0 \mu \mathrm{M} \mathrm{SeO}_{3}{ }^{2-}$ as necessary for maximizing the number of observed cfus. We hypothesize that such a supplementation is necessary because the overexpression of a $\mathrm{SeO}_{3}{ }^{2-}$ - reducing enzyme in these cells reduces and makes unavailable the essential amount of Se needed as a micronutrient for optimum growth. ${ }^{30}$

Figure 2 shows relative growth inhibition as a function of selenite concentration. The $\log _{10}\left[\mathrm{SeO}_{3}{ }^{2-}\right]$ gives a linear concentration dependence for $\mathrm{SeO}_{3}{ }^{2-}$ growth inhibition. This allows the determination of the inhibitory concentration of selenite that eliminates $90 \%$ of cell growth $\left(\mathrm{IC}_{90}\right)$. We found an $\mathrm{IC}_{90}$ of $21.3 \pm 9.80 \mathrm{mM}$ under the conditions of GRLMR overexpression, whereas an $\mathrm{IC}_{90}$ of $1.89 \pm 0.46 \mathrm{mM}$ is observed in a corresponding control experiment. This result is at least somewhat cell-line-independent. When GRLMR is recombinantly overexpressed in E. coli SS320, we observe an $\mathrm{IC}_{90}$ of $18.3 \pm 19.50 \mathrm{mM}$. GRLMR scavenges the cells of the micronutrient levels of selenium which can stunt growth and cause cell death. This inherently causes large deviations in cfu formation from run to run. Even with the large error bars, a statistically significant increase in $\mathrm{SeO}_{3}{ }^{2-}$ tolerance is induced by the presence of the GRLMR plasmid, and we believe that the SS320 data is justified because of the similarly observed tolerance in the BL21 cell line.

We note that the presence of the recombinantly expressed enzyme results in the liquid cultures taking on the red color characteristic of the red allotrope of zerovalent selenium, whereas cultures grown with $\mathrm{SeO}_{3}{ }^{2-}$ without the recombinantly expressed enzyme do not take on this color. To illustrate this, Figure 3 shows the cell cultures expressing GRLMR (left panel) or the green fluorescent protein (GFP) (right panel) in the presence of $\mathrm{SeO}_{3}{ }^{2-}$ supplementation at 5.0 $\mathrm{mM}$, after $3 \mathrm{~h}$ of exposure. This "bulk color" change suggests that the cells expressing the recombinant enzyme may also be forming $\mathrm{Se}(0) \mathrm{NPs}$, just as we previously observed for the $P$. moravenis strain.

The examination of cells by scanning electron microscopy (SEM) revealed the presence of selenium NPs in cells expressing the recombinant enzyme and grown in Sesupplemented media. Figure 4 shows the scanning transmission electron micrographs of glutaraldehyde-fixed drymounted BL21 E. coli cells expressing GFP or GRLMR after growth in $\mathrm{SeO}_{3}{ }^{2-}$-supplemented media. Both GFP and GRLMR cells show dark inclusions, with more inclusions observed in the GRLMR-expressing cell line. The dark

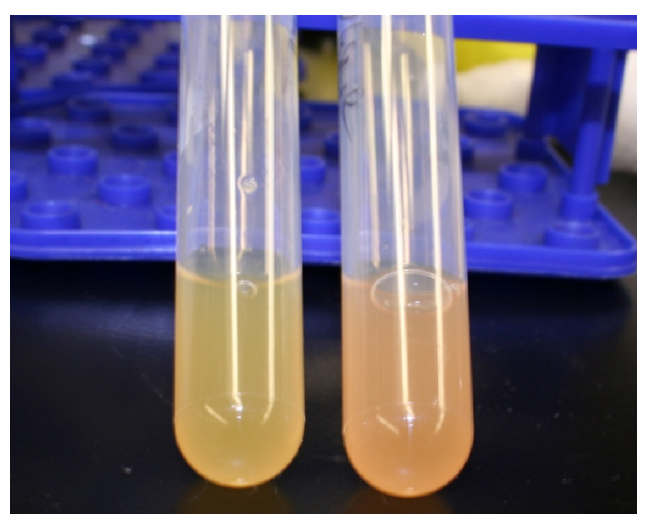

Figure 3. Separation of selenium NP growth. BL21 cells with (right) and without (left) the GRLMR plasmid after a $3 \mathrm{~h}$ exposure to 5.0 $\mathrm{mM}$ selenite-supplemented LB.
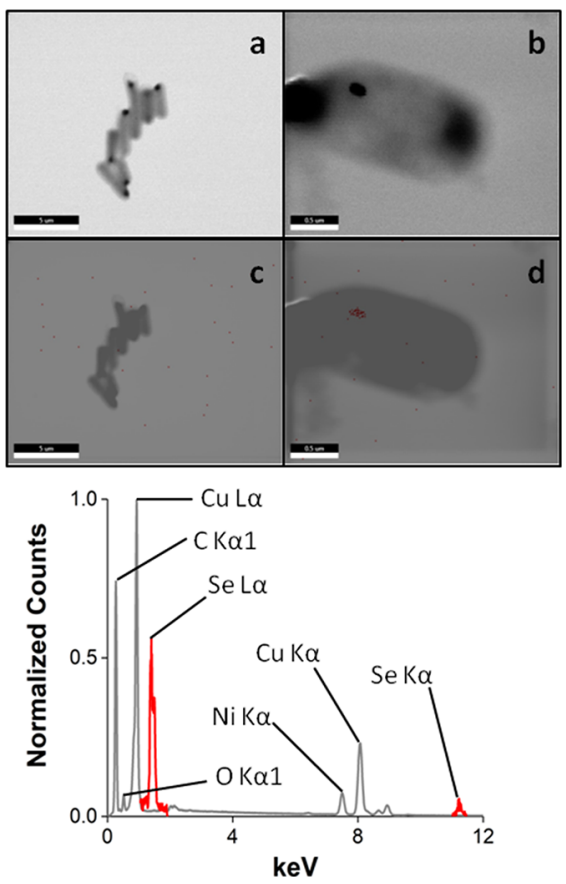

Figure 4. SEM images (a,b) with EDS overlay $(c, d)$ of fixed cells after $3 \mathrm{~h}$ exposure to $5 \mathrm{mM}$ selenite. $(\mathrm{a}, \mathrm{c})$ Cells without the GRLMR plasmid. Scale bars are $5 \mu \mathrm{m}$. (b,d) Cells with the GRLMR plasmid. Scale bars are $0.5 \mu \mathrm{m}$. The corresponding EDS spectra of cells without GRLMR (gray) and additional peaks from cells with the GRLMR plasmid (red) on bottom. Cu peaks are present in our sample, as carbon-coated $\mathrm{Cu}$ mesh grids were used. 
Table 3. Key Glutathione Pocket Residues

\begin{tabular}{ccccc} 
enzyme & PM MTLR & PF GSHR & EC GSHR & SC GSHR \\
key product-binding site residues & $\alpha$-Ser & $\alpha$-Ser & $\alpha$-Met & $\beta$ L-Lys \\
& $\beta$ L-Lys & $\beta$ L-Lys & $\beta$-Met & $\beta$ R-Lys \\
& $\beta$ R-Glu & $\beta$ R-Glu & $\beta$ R-Asn \\
\hline
\end{tabular}

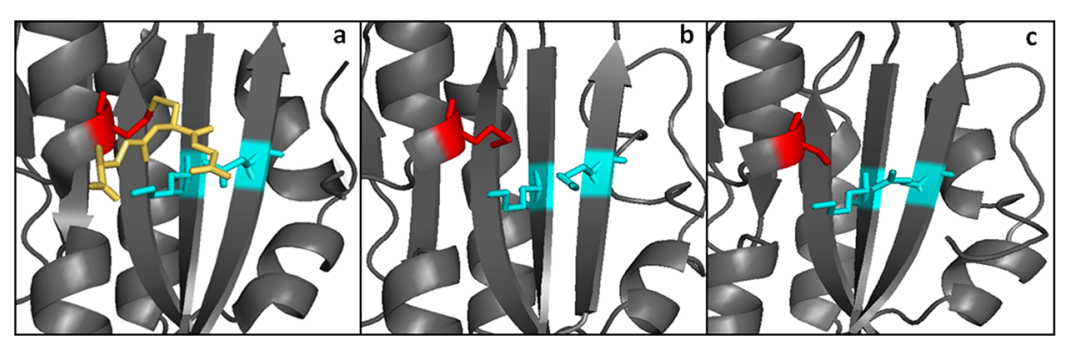

Figure 5. Red $--\alpha$ residue, teal $--\beta$ residues, and yellow--GSH. (a) Yeast GHSR substrate-binding pocket with bound GSH. (b) E. coli GSHR substrate-binding pocket. (c) GRLMR substrate-binding pocket.

inclusions seen in both cell lines near the cell walls are most likely the cellular nuclei because of their cellular placement and single appearance per cell. Electron-dispersive spectroscopy (EDS) mapping confirms that the dark inclusions are Se-rich for the GRLMR cells, whereas any inclusions observed in the GFP-expressing cells show no evidence of Se presence. The corresponding EDS spectra further confirm these conclusions by the presence of signature selenium peaks being present for the GRLMR cells and the absence of these peaks for the GFP cells. Complete EDS mapping for the control and GRLMR cells can be seen in Figure S6. The intracellular Se-rich NPs have an amorphous surface similar to our previously reported results, ${ }^{13}$ suggesting a partial cytosol-exposed surface. This appears to be unique to our enzyme-synthesized particles because inorganic/bioinorganic methods tend to make smooth-surfaced particles and require capping agents for stabilization..$^{31,32}$ The transportability of enzymatic function to foreign cell lines further demonstrates the ability for this enzyme's potential application as a clonable NP contrast generator.

Previously, we observed that some fraction of NPs synthesized by $S$. cervisiae GSHR were associated with the particle fraction. ${ }^{13}$ We examined GRLMR for a similar behavior. Selenium particles were synthesized in vitro and were separated from the solution using centrifugation. The analysis of the protein content in the solution and the particles were determined by Bradford assay (Bio-Rad). Overall, approximately $10 \%$ of the enzyme is associated with the selenium NPs. This is a smaller fraction than we observed for S. cervisiae GSHR $(\sim 18 \%)$, suggesting that the P. moravenisderived enzyme is more efficient at turning over or releasing the particles that they create. Overall, this contributes to the picture of GRLMR being specialized for conveying $\mathrm{Se}$ tolerance.

Differences in the product-/substrate-binding pocket of this family of enzymes may underlie any observed differences in substrate specificity and enzyme activity. The key residues in the product-/substrate-binding areas of GSHR are shown in Table 3, and the models of these are shown in Figure S3. The structural alignment between GRLMR and $P$. fluorescines reveals an RMSD of $1.71 \AA$ for the full enzyme and $1.56 \AA$ for the product-/substrate-binding pocket. These values show the largest deviation between any of the enzymes considered here, but to the best of our knowledge, no crystal structure of
Pseudomonas GSHR has been obtained which would affect the generated structure models.

The product-/substrate-binding pocket for GSHR contains a set of evolutionarily conserved residues, most notably including a cysteine that is implicated in glutathionylation regulatory mechanisms. There are three residues that dominate the binding interaction, one on an $\alpha$-helix, and two on parallel $\beta$-strands $(\beta \mathrm{L}, \beta \mathrm{R})$. Comparing our Pseudomonas enzymes, we see that they contain the same key residues; $\alpha$-Ser, $\beta \mathrm{L}$-Lys, and $\beta$ R-Glu (Figure 5). ${ }^{33}$

This absence of a sulfur-containing residue (Figure 1) in the P. moravenis GRLMR suggests that the enzyme is not subject to the glutathionylation regulatory mechanism well-established for canonical GSHR enzymes such as S. cervisiae GSHR. Glutathionylation of enzymes is a common post-translation modification for proteins in signaling pathways and survival gene modification. ${ }^{34-37}$ This reversible post-translation modification is the binding of glutathione to an unpaired cysteine residue. ${ }^{38}$ Such a modification alters the enzyme activity, presumably as a regulation mechanism. ${ }^{39,40}$ In the case of $S$. cervisiae GSHR, glutathionylation at C239 (Figure 5) inhibits the enzyme. Chemically blocking the glutathionylation pathway is shown to increase the GSHR activity by a factor of 2.1. ${ }^{41}$ Overall, the absence of the possibility of a glutathionylation regulation mechanism for the GRLMR enzyme suggests that it is distinct from the other GSHR enzymes.

The selenium metabolism literature highlights several examples of species within the Pseudomonas genus with remarkable tolerance to Se. In many cases, GSHR enzymes are implicated in the tolerance. GSHR was responsible for reducing selenite and tellurite to insoluble NPs using the O-2 strain of Pseudomonas maltophilia; ${ }^{42}$ GSHR and thioredoxin reductase are responsible for selenite and selenate reduction in Pseudomonas seleniipraecipitansI. ${ }^{43-46}$ The highly conserved sequence across species within the Pseudomonas genus, including conservation in the product/substrate binding pocket, is suggestive that the ability to handle the normally toxic amounts of $\mathrm{SeO}_{3}{ }^{2-}$ may be a general feature of the Pseudomonas genus. This Se tolerance may arise from the nature of GSHRs in this genus. 


\section{CONCLUSIONS}

In summary, we have characterized GRLMR from the bacterium Pseudomonas moraviensis stanleyae. The kinetic studies showed an overall decrease in substrate affinity for GRLMR relative to the $S$. cervisiae GSHR, but an overall increased affinity for GS-Se-SG over GSSG. The transportability of the gene was tested by transforming the lab strain E. coli with GRLMR. Selenite tolerance increased 10-fold compared to the cells without the gene, and elemental red selenium was formed when GRLMR was present. SEM/EDS further confirmed this by showing the selenium particles associated with the cells containing the gene. Product association experiments showed a decrease in product retention when compared to the $S$. cervisiae GSHR, which ultimately allows for increased product release and contributes to the overall Se tolerance.

\section{MATERIALS AND METHODS}

Identification/Isolation. An LB agar plate with colonies of the original $P$. moravenis cell line was submitted for fullgenome sequencing. The DNA sequence from the most closely related enzyme identified by MALDI-MS was used to identify the sequence of our enzyme of interest. This sequence was cloned into a $\mathrm{pD} 441-\mathrm{CH}$ E. coli vector, and a standard heatshock protocol was used to transform BL21 E. coli.

Standard protein purification was conducted by growing cells in $1 \mathrm{~L}$ of $\mathrm{LB}$ to an optical density at $600 \mathrm{~nm}\left(\mathrm{OD}_{600}\right)$ of 0.6 and inducing protein expression with $1 \mathrm{mM}$ IPTG for $2 \mathrm{~h}$. The cells were collected and resuspended in $25 \mathrm{~mL}$ of lysis buffer and lysed by tip sonication. The soluble cell lysate was collected and nickel-agarose beads were used to isolate and wash our expressed protein.

GS-Se-SG Synthesis. The protocol from Ganther was followed for the synthesis of selenodiglutathione. ${ }^{26}$ To $24 \mathrm{~mL}$ of $0.1 \mathrm{M} \mathrm{HCl}, 400$ moles of $\mathrm{HNaSeO}_{3}$ was added and cooled to $4{ }^{\circ} \mathrm{C}$. Another solution of $0.1 \mathrm{M} \mathrm{GSH}$ was cooled to $4{ }^{\circ} \mathrm{C}$ and added quickly to the selenite solution. The mixture was allowed to react at $4{ }^{\circ} \mathrm{C}$ for $20 \mathrm{~min}$. A $2.5 \mathrm{~mL}$ of $2 \mathrm{M} \mathrm{NaOAc}$ was added to obtain a final $\mathrm{pH}$ of 4.5 . A C18 column was used to separate the products using $\mathrm{pH} 2.0 \mathrm{HCl}$. Thin-layer chromatography was used to check the contents of the lyophilized fractions. The isolated GS-Se-SG was identified and the amount quantified using UV-vis absorption at $263 \mathrm{~nm}$. (S.4)

Km. $1 \mathrm{~mL}$ reactions were conducted in $1 \times$ phosphatebuffered saline with $0.1 \mathrm{mM} \mathrm{NADPH}$ and $15 \mu \mathrm{g}$ of either purified enzyme or S. cervisiae GSHR purchased from Sigma. The substrates tested were GSSG, GS-Se-SG, and selenite. Their concentrations were varied between reactions, and the depletion of the NADPH peak at $340 \mathrm{~nm}$ was monitored every $2 \mathrm{~s}$ after the contents were mixed.

Transportability. $\mathrm{IC}_{90}$ 's were determined by standard plating experiments. In short, cultures of BL21 cells containing either a plasmid with GRLMR or a generic reporter gene were grown overnight in $\mathrm{LB}$ at $37^{\circ} \mathrm{C}$. The following morning, 100 $\mu \mathrm{L}$ of this starter culture was added to $2.5 \mathrm{~mL}$ of fresh $\mathrm{LB}$ and grown for roughly $2.5 \mathrm{~h}$ to reach an $\mathrm{OD}_{600}$ of 0.6 . Various amounts of selenite were added to each culture, and exposure was continued for $24 \mathrm{~h}$. After the exposure, the cells were diluted $10^{6}$-fold, and $20 \mu \mathrm{L}$ of each dilution was plated in triplicate on $1 \times$ Kanamycin LB agar. The plates were put in an oven at $37^{\circ} \mathrm{C}$, and colonies were grown overnight and counted the following day.

Scanning Transmission Electron Microscopy (STEM). A volume of $3 \mathrm{~mL}$ of BL21 cells containing either a metalloid reductase gene or GFP reporter gene was grown separately in $10 \mathrm{~mL}$ culture tubes overnight containing LB medium (Teknova) supplemented with Kanamycin at $25 \mu \mathrm{g} / \mathrm{mL}$. The following morning, the culture was added to a $125 \mathrm{~mL}$ Erlenmeyer flask containing LB medium supplemented with Kanamycin $(25 \mu \mathrm{g} / \mathrm{mL})$. The cells were grown for $2.5 \mathrm{~h}$, and $100 \mathrm{mM} \mathrm{Na}_{2} \mathrm{SeO}_{3}$ (Alfa Aesar, 98+\%) was added to reach a final concentration of $5 \mathrm{mM}$. The cells were collected by centrifuging for $20 \mathrm{~min}$ at $4000 \mathrm{rpm}$ and $4{ }^{\circ} \mathrm{C}$ after $3 \mathrm{~h}$ of growth with selenite. The cells were washed with $20 \mathrm{mM}$ Tris ( $\mathrm{pH} 7.4)$ (Fischer) three times followed by resuspension in 1 $\mathrm{mL}$ of fixing solution ( $2 \%$ glutaraldehyde ( $25 \%$ Sigma-Aldrich) and $2.5 \%$ formaldehyde); the fixing solution was allowed to react for $12 \mathrm{~h}$ at $4{ }^{\circ} \mathrm{C}$. The fixing solution was centrifuged and the pellet was washed five times in $20 \mathrm{mM}$ Tris ( $\mathrm{pH} \mathrm{7.4).} \mathrm{The}$ cells were resuspended in $1 \mathrm{~mL}$ of $20 \mathrm{mM}$ Tris ( $\mathrm{pH}$ 7.4). The aliquots $(4 \mu \mathrm{L})$ were mounted on 400 mesh $\mathrm{Cu}$ grids with 50 $\mathrm{nm} \mathrm{C}$ coating and washed two times with $\mathrm{H}_{2} \mathrm{O}$. The drymounted cells on transmission electron microscopy grids were loaded onto a STEM holder. The STEM images were taken with a JEOL JSM-6500-F scanning electron microscope at an accelerating voltage of $15 \mathrm{kV}$.

EDS. EDS was performed on the P. moreviensis stanleyae cells with SEM, as described above. EDS was collected on a NORAN System 7 X-ray microanalysis detector with a time interval of $1 \mathrm{~s}$.

\section{ASSOCIATED CONTENT}

\section{Supporting Information}

The Supporting Information is available free of charge on the ACS Publications website at DOI: 10.1021/acsomega.8b00826.

Genome sequencing scores, sequence alignment, generated model templates, glutathione-binding sites, GSSe-SG UV-vis, $K_{\mathrm{m}}$ assay UV-vis, and SEM/EDS (PDF)

\section{AUTHOR INFORMATION}

\section{Corresponding Author}

*E-mail: ackerson@colostate.edu.

ORCID

Christopher J. Ackerson: 0000-0001-6863-6054

\section{Present Addresses}

${ }^{\dagger}$ Sensient Colors, St. Louis, Missouri 63106, United States. ثniversity of Texas Southwestern Medical Center, Department of Cell Biology, Dallas, Texas 75390, United States.

\section{Author Contributions}

The manuscript was written through contributions of all authors. All authors have given approval to the final version of the manuscript.

\section{Funding}

NIH R01 GM112225 A1. NSF CAREER 1455099.

\section{Notes}

The authors declare no competing financial interest. 


\section{ACKNOWLEDGMENTS}

The authors thank Zachary Butz for many conversations on technical aspects of this work.

\section{ABBREVIATIONS}

DPS, DNA-binding protein; GSHR, glutathione reductase; NADPH, nicotinamide adenine dinucleotide phosphate; GRLMR, glutathione reductase-like-metalloid reductase; BLAST, basic local alignment search tool; RMSD, rootmean-square deviation; NP, nanoparticle; IPTG, isopropyl $\beta$-D1-thiogalactopyranoside; cfus, colony-forming units; GFP, green fluorescent protein; SEM, scanning electron microscopy; EDS, electron-dispersive spectroscopy; LB, lysogeny broth; MALDI-MS, matrix-assisted laser desorption/ionization-mass spectrometry; OD, optical density; TLC, thin-layer chromatography; PBS, phosphate-buffered saline

\section{REFERENCES}

(1) Harrison, P. M. The structure and function of ferritin. Biochem. Educ. 1986, 14, 154-162.

(2) Kolinko, I.; et al. Biosynthesis of magnetic nanostructures in a foreign organism by transfer of bacterial magnetosome gene clusters. Nat. Nanotechnol. 2014, 9, 193-197.

(3) Müller, W. E. G.; et al. Silicateins, the major biosilica forming enzymes present in demosponges: Protein analysis and phylogenetic relationship. Gene 2007, 395, 62-71.

(4) Carter, C. J.; et al. In vitro selection of RNA sequences capable of mediating the formation of iron oxide nanoparticles. J. Mater. Chem. 2009, 19, 8320-8326.

(5) Slocik, J. M.; Kuang, Z.; Knecht, M. R.; Naik, R. R. Optical Modulation of Azobenzene-Modified Peptide for Gold Surface Binding. ChemPhysChem 2016, 17, 3252-3259.

(6) Yeh, H.-C.; Sharma, J.; Han, J. J.; Martinez, J. S.; Werner, J. H. A DNA-Silver Nanocluster Probe That Fluoresces upon Hybridization. Nano Lett. 2010, 10, 3106-3110.

(7) Klug, M. T.; et al. Mediated Growth of Zinc Chalcogen Shells on Gold Nanoparticles by Free-Base Amino Acids. Chem. Mater. 2017, 29, 6993-7001.

(8) Choi, S.; Dickson, R. M.; Lee, J.-K.; Yu, J. Generation of luminescent noble metal nanodots in cell matrices. Photochem. Photobiol. Sci. 2012, 11, 274-278.

(9) Wang, Q.; Mercogliano, C. P.; Löwe, J. A ferritin-based label for cellular electron cryotomography. Struct. 2011, 19, 147-154.

(10) Castruita, M.; et al. Overexpression and Characterization of an Iron Storage and DNA-Binding Dps Protein from Trichodesmium erythraeum. Appl. Environ. Microbiol. 2006, 72, 2918-2924.

(11) Fox, B.; Walsh, C. T. Mercuric reductase. Purification and characterization of a transposon-encoded flavoprotein containing an oxidation-reduction-active disulfide. J. Biol. Chem. 1982, 257, 24982503.

(12) Reed, J. K. Studies on the kinetic mechanism of lipoamide dehydrogenase from rat liver mitochondria. J. Biol. Chem. 1973, 248, 4834-4839.

(13) Ni, T. W.; et al. Progress toward clonable inorganic nanoparticles. Nanoscale 2015, 7, 17320-17327.

(14) Lian, P.; et al. X-ray Structure of a Hg2+ Complex of Mercuric Reductase (MerA) and Quantum Mechanical/Molecular Mechanical Study of $\mathrm{Hg} 2+$ Transfer between the C-Terminal and Buried Catalytic Site Cysteine Pairs. Biochemistry 2014, 53, 7211-7222.

(15) Fox, B. S.; Walsh, C. T. Mercuric reductase: homology to glutathione reductase and lipoamide dehydrogenase. Iodoacetamide alkylation and sequence of the active site peptide. Biochemistry 1983, 22, 4082-4088.

(16) Li, X.; Krumholz, L. R. Thioredoxin Is Involved in U(VI) and $\mathrm{Cr}(\mathrm{VI})$ Reduction in Desulfovibrio desulfuricans G20. J. Bacteriol. 2009, 191, 4924-4933.
(17) Freedman, Z.; Zhu, C.; Barkay, T. Mercury Resistance and Mercuric Reductase Activities and Expression among Chemotrophic Thermophilic Aquificae. Appl. Environ. Microbiol. 2012, 78, 65686575.

(18) Pugin, B.; et al. Glutathione Reductase-Mediated Synthesis of Tellurium-Containing Nanostructures Exhibiting Antibacterial Properties. Appl. Environ. Microbiol. 2014, 80, 7061-7070.

(19) Otwell, A. E.; et al. Identification of proteins capable of metal reduction from the proteome of the Gram-positive bacterium D esulfotomaculum reducens MI-1 using an NADH-based activity assay. Environ. Microbiol. 2015, 17, 1977-1990.

(20) Scott, D.; Toney, M.; Muzikár, M. Harnessing the mechanism of glutathione reductase for synthesis of active site bound metallic nanoparticles and electrical connection to electrodes. J. Am. Chem. Soc. 2008, 130, 865-874.

(21) Wang, Z.; et al. De novo assembly and characterization of root transcriptome using Illumina paired-end sequencing and development of cSSR markers in sweetpotato (Ipomoea batatas). BMC Genomics 2010, 11, 726 .

(22) Ewing, B.; Hillier, L.; Wendl, M. C.; Green, P. Base-Calling of Automated Sequencer Traces UsingPhred. I. Accuracy Assessment. Genome Res. 1998, 8, 175-185.

(23) Arscott, L. D.; Gromer, S.; Schirmer, R. H.; Becker, K.; Williams, C. H. The mechanism of thioredoxin reductase from human placenta is similar to the mechanisms of lipoamide dehydrogenase and glutathione reductase and is distinct from the mechanism of thioredoxin reductase from Escherichia coli. Proc. Natl. Acad. Sci. U.S.A. 1997, 94, 3621-3626.

(24) Mittl, P. R. E.; Schulz, G. E. Structure of glutathione reductase from Escherichia coli at 1.86 A resolution: comparison with the enzyme from human erythrocytes. Protein Sci. 2008, 3, 799-809.

(25) Pai, E. F.; Schulz, G. E. The catalytic mechanism of glutathione reductase as derived from $\mathrm{x}$-ray diffraction analyses of reaction intermediates. J. Biol. Chem. 1983, 258, 1752-1757.

(26) Ganther, H. E. Reduction of the selenotrisulfide derivative of glutathione to a persulfide analog by gluthathione reductase. Biochemistry 1971, 10, 4089-4098.

(27) Kelley, L. A.; Mezulis, S.; Yates, C. M.; Wass, M. N.; Sternberg, M. J. E. The Phyre2 web portal for protein modeling, prediction and analysis. Nat. Protoc. 2015, 10, 845-858.

(28) Mavis, R. D.; Stellwagen, E. Purification and Subunit Structure of Glutathione Reductase from Bakers' Yeast. J. Biol. Chem. 1968, 243, 809-814.

(29) Painter, E. P. The Chemistry and Toxicity of Selenium Compounds, with Special Reference to the Selenium Problem. Chem. Rev. 1941, 28, 179-213.

(30) Hunter, P. A toxic brew we cannot live without. Micronutrients give insights into the interplay between geochemistry and evolutionary biology. ЕMBO Rep. 2008, 9, 15-18.

(31) Nath, S.; Ghosh, S. K.; Panigahi, S.; Thundat, T.; Pal, T. Synthesis of Selenium Nanoparticle and Its Photocatalytic Application for Decolorization of Methylene Blue under UV Irradiation. Langmuir 2004, 20, 7880-7883.

(32) Ramamurthy, C. H.; et al. Green synthesis and characterization of selenium nanoparticles and its augmented cytotoxicity with doxorubicin on cancer cells. Bioproc. Biosyst. Eng. 2013, 36, 11311139.

(33) Yu, J.; Zhou, C.-Z. Crystal structure of glutathione reductase Glr1 from the yeast Saccharomyces cerevisiae. Proteins: Struct., Funct., Bioinf. 2007, 68, 972-979.

(34) Grek, C. L.; Zhang, J.; Manevich, Y.; Townsend, D. M.; Tew, K. D. Causes and Consequences of CysteineS-Glutathionylation. J. Biol. Chem. 2013, 288, 26497-26504.

(35) Sullivan, D. M.; Wehr, N. B.; Fergusson, M. M.; Levine, R. L.; Finkel, T. Identification of Oxidant-Sensitive Proteins: TNF- $\alpha$ Induces Protein Glutathiolation. Biochemistry 2000, 39, 1112111128 . 
(36) Townsend, D. M.; et al. A glutathione S-transferase pi-activated prodrug causes kinase activation concurrent with S-glutathionylation of proteins. Mol. Pharmacol. 2006, 69, 501-508.

(37) Fiaschi, T.; et al. Redox Regulation of $\beta$-Actin during Integrinmediated Cell Adhesion. J. Biol. Chem. 2006, 281, 22983-22991.

(38) Hill, B. G.; Bhatnagar, A. Protein S-glutathiolation: Redoxsensitive regulation of protein function. J. Mol. Cell. Cardiol. 2012, 52, $559-567$.

(39) Humphries, K. M.; Juliano, C.; Taylor, S. S. Regulation of cAMP-dependent protein kinase activity by glutathionylation. J. Biol. Chem. 2002, 277, 43505-43511.

(40) Townsend, D. M.; et al. Nitrosative stress-induced sglutathionylation of protein disulfide isomerase leads to activation of the unfolded protein response. Cancer Res. 2009, 69, 7626-7634.

(41) Stepovaya, E. A.; et al. The role of oxidative protein modification and the glutathione system in modulation of the redox status of breast epithelial cells. Biochem. Mosc. Suppl. Ser. B Biomed. Chem. 2016, 10, 235-239.

(42) Blake, R. C.; et al. Chemical transformation of toxic metals by aPseudomonasstrain from a toxic waste site. Environ. Toxicol. Chem. 1993, 12, 1365-1376.

(43) Hunter, W. J.; Manter, D. K. Reduction of selenite to elemental red selenium by Pseudomonas sp. Strain CA5. Curr. Microbiol. 2009, 58, 493-498.

(44) Kora, A. J.; Rastogi, L. Biomimetic synthesis of selenium nanoparticles by Pseudomonas aeruginosa ATCC 27853: An approach for conversion of selenite. J. Environ. Manage. 2016, 181, 231-236.

(45) Lortie, L.; Gould, W. D.; Rajan, S.; McCready, R. G. L.; Cheng, K.-J. Reduction of Selenate and Selenite to Elemental Selenium by a Pseudomonas stutzeri Isolate. Appl. Environ. Microbiol. 1992, 58, 4042-4044.

(46) Avendaño, R.; et al. Production of selenium nanoparticles in Pseudomonas putida KT2440. Sci. Rep. 2016, 6, 37155. 